Ann. Biol. anim. Bioch. Biophys., I972, 12 (3), 493-504.

\title{
CHROMATOGRAPHIE EN PHASE GAZEUSE DES OSES, DES DI- ET TRIHOLOSIDES DANS LES MILIEUX COMPLEXES
}

\author{
J.-P. JOUANY \\ Station de Recherches sur l'Élevage des Ruminants, \\ Centre de Recherches de Clermont-Ferrand, I. N.R. A., \\ Theix, 63 - Saint-Genès-Champanelle
}

\section{RÉSUMÉ}

La méthode que nous proposons permet de séparer et de doser simultanément les pentoses, les hexoses, les di- et triholosides à l'état libre ou obtenus après hydrolyse, dans les milieux complexes.

Les glucides présents dans l'échantillon sec à analyser sont transformés en dérivés triméthylsilylés avant d'être injectés en tête de colonne. L'utilisation d'un standard interne (mésoinositol) permet d'évaluer quantitativement les différents pics obtenus.

Les modifications que nous avons apportées aux méthodes classiques de chromatographie en phase gazeuse des glucides sont les suivantes:

- La silylation s'effectue dans le diméthylformamide (ELLIs, 1969) et non dans la pyridine, ce qui permet d'obtenir des résultats quantitatifs plus précis surtout dans le domaine d'élution des pentoses.

- Les quantités des réactifs de silylation (hexaméthyldisilazane et triméthylchlorosilane) ont été déterminées de façon à doser quantitativement les glucides même si l'échantillon n'est pas parfaitement anhydre.

- La phase stationnaire utilisée $\left(\mathrm{OV}_{1}\right)$ a une température limite d'utilisation élevée $\left(35^{\circ} \mathrm{C}\right)$, ce qui permet l'élution des triholosides à $320^{\circ} \mathrm{C}$.

Cette méthode permet une séparation correcte de la plupart des glucides simples que l'on rencontre généralement dans les milieux biologiques. Cependant, certains couples de glucides sont mal séparés et la durée d'analyse de l'ensemble des sucres est relativement longue.

\section{INTRODUCTION}

Le dosage des glucides par chromatographie en phase gazeuse est une méthode récente qui est plus spécifique, plus rapide et plus précise que la colorimétrie ou que les autres méthodes chromatographiques. Depuis les travaux de Mac InNes et al. (I958), les auteurs qui se sont intéressés à cette technique ont étudié, soit les diffé- 
rentes formes des dérivés volatils que l'on peut utiliser (éthers méthylés ou silylés, esters acétylés, alditols estérifiés, etc.), soit la préparation de nouvelles colonnes chromatographiques. Plusieurs méthodes ont été proposées pour la séparation de certaines catégories de glucides (tabl. I). Cependant, peu de travaux ont été entrepris pour séparer simultanément et de façon satisfaisante les pentoses, les hexoses, les di- et triholosides. Nous pensons avoir résolu ce problème et proposons une méthode qui sépare ces différents glucides à partir d'un même chromatogramme. Nos conditions opératoires permettent d'appliquer cette méthode à des produits contenant de faibles quantités de glucides dans la prise d'essai (de $0, \mathrm{I} \mathrm{mg}$ à I mg).

\section{TABLEAU I}

Données bibliographiques concernant les principales méthodes de dosage des glucides par chromatographie en phase gazeuse

\begin{tabular}{|c|c|c|c|}
\hline Auteurs & Sucres séparés & Dérivés volatils & Produits étudiés \\
\hline MAC Innes et al. (1958) $\ldots \ldots \ldots \ldots \ldots \ldots$ & $\begin{array}{l}\text { Pentoses } \\
\text { et hexoses }\end{array}$ & Dérivés méthylés & Sucres purs \\
\hline Bishop et Cooper $(1960) \ldots \ldots \ldots \ldots \ldots$ & $\begin{array}{l}\text { Pentoses } \\
\text { et hexoses }\end{array}$ & Dérivés acétylés & - \\
\hline HEDGLEy et OVERENd $(1960) \ldots \ldots \ldots \ldots$ & $\begin{array}{l}\text { Hexoses, di- } \\
\text { et triholosides }\end{array}$ & $\begin{array}{l}\text { Dérivês triméthyl- } \\
\text { silylés (TMS) }\end{array}$ & 一 \\
\hline SWEELEY et al. (1963) & - & - & - \\
\hline SAWARDEKER, SLONEKER et JEANES (1965) ... & $\begin{array}{l}\text { Pentoses } \\
\text { et hexoses }\end{array}$ & Alditols acétates & 一 \\
\hline Wells, Chin et Weber (1964) . . . . . . . . & Hexoses & Dérivés TMS & $\begin{array}{l}\text { Urine et sérum } \\
\text { sanguin }\end{array}$ \\
\hline BRoBst et Lot (1966) $\ldots \ldots \ldots \ldots \ldots \ldots$ & $\begin{array}{l}\text { Glucose, maltose, } \\
\text { maltotriose } \\
\text { et maltotétraose }\end{array}$ & & Sirop de mais \\
\hline Oates et Schrager $(1967) \ldots \ldots \ldots$ & $\begin{array}{c}\text { Pentoses } \\
\text { et hexoses }\end{array}$ & - & $\begin{array}{l}\text { Hydrolysats de } \\
\text { mucopoly- } \\
\text { saccharides } \\
\text { gastriques } \\
\text { et salivaires }\end{array}$ \\
\hline Clapperton et Holliday $(1968) \ldots \ldots \ldots \ldots$ & $\begin{array}{l}\text { Hexoses, di- tri- } \\
\text { et tétraholosides }\end{array}$ & 一 & Moût de bière \\
\hline KIMURA et al., (1969) $\ldots \ldots \ldots \ldots \ldots \ldots \ldots$ & $\begin{array}{l}\text { Hexoses, di- } \\
\text { et tribolosides }\end{array}$ & - & $\begin{array}{c}\text { Sucres de la } \\
\text { pomme de terre }\end{array}$ \\
\hline
\end{tabular}

\section{MÉTHODE PROPOSÉE}

\section{Principe}

Les échantillons anhydres sont analysés directement, tandis que les échantillons humides sont lyophilisés. Ils subissent ensuite un traitement chimique qui transforme les fonctions hydroxyles des glucides en éthers $O$-triméthylsilylés qui sont volatils. Ceux-ci sont ensuite séparés par la technique de chromatographie en phase gazeuse. L'utilisation d'un standard interne (mésoinositol) permet d'évaluer quantitativement les différents pics obtenus. 


\section{Chromatographe.}

\section{Appareillage}

Le chromatographe utilisé est un appareil Girdel $75 \mathrm{CD}$. PT à double colonne, avec programmation de température (Giravions Dorand), muni de deux détecteurs à ionisation de flamme (Nunnikoven, I968). L'enregistreur "Servoriter II " (Texas Instruments Incorporation) de sensibilité $\mathrm{I} \mathrm{mV}$ et de temps de réponse 0,4 seconde, est équipé d'un intégrateur mécanique «Disc .

\section{Colonnes.}

Les deux colonnes, de diamètre intérieur $2,17 \mathrm{~mm}$ et de diamètre extérieur $3,18 \mathrm{~mm}$, sont en acier inoxydable. Chaque colonne contient environ 2,3 grammes de Varaport 30 (IOo I20 mesh) (Varian Aerograph), imprégné de $O_{1}$, à raison de 3 grammes pour Ioo grammes de support. Une seule colonne est utilisée pour l'analyse des échantillons (colonne analytique), l'autre sert de colonne de référence afin de corriger la dérive de la ligne de base au cours de la programmation de température.

Débit des gaz.

Le gaz vecteur est l'azote. Son débit optimum est déterminé pour chaque colonne en traçant la courbe de VAN DeEmTer (1956) qui montre la variation de la hauteur d'un plateau théorique en fonction de la vitesse linéaire $(\bar{u})$ du gaz vecteur à la sortie de la colonne:

$$
\mathrm{H}=\mathrm{A}+\frac{\mathrm{B}}{\bar{u}}+\mathrm{C} \bar{u}
$$

Les colonnes que nous avons utilisées ont un débit optimum d'environ 22,5 ml/mn.

La combustion au niveau du détecteur est assurée par un mélange d'hydrogène et d'air.

Le débit est de $20 \mathrm{ml} / \mathrm{mn}$ pour l'hydrogène et $400 \mathrm{ml} / \mathrm{mn}$ pour l'air.

\section{Caractéristiques des colonnes}

La pression d'entrée (Pe) sur les colonnes est de $\mathrm{I}, 45$ bar à $\mathrm{r} 40^{\circ} \mathrm{C}$, tandis que la pression de sortie (Ps) correspond à la pression atmosphérique. Le facteur de correction de la compressibllité $J$ est calculé suivant la formule :

$$
\mathrm{J}=\frac{3}{2} \times \frac{\mathrm{P}^{2}-\mathrm{I}}{\mathrm{P}^{3}-\mathrm{I}} \quad \text { avec } \quad \mathrm{P}=\frac{\mathrm{P}_{\mathrm{e}}}{\mathrm{P}_{\mathrm{s}}} ; \mathrm{J}=0,76 \text { à } \mathrm{I}_{4} 0^{\circ} \mathrm{C}
$$

Le volume de rétention spécifique des colonnes a été déterminé pour l'alpha-xylose ;

$$
\mathrm{V}=\mathrm{J} \times \mathrm{D} s \times(d r-d m) \times \frac{273}{\mathrm{U}_{\mathbf{e}} \mathrm{Mg} \mathrm{T}_{\mathrm{c}}}=0,36 \mathrm{I} \mathrm{ml} / \mathrm{mg}
$$

Nous avons en outre déterminé le nombre des plateaux théoriques pour quelques composés couramment rencontrés au cours des analyses (tabl. 2).

\section{Réactifs de silylation}

Le solvant utilisé pour dissoudre les sucres est le diméthylformamide. Les réactifs de silylation sont l'hexaméthyldisilazane et le triméthylchlorosilane. Le catalyseur éventuel est l'acide trifluoracétique.

\section{MODE OPÉRATOIRE}

\section{Préparation des dérivés volatils}

On pèse une partie aliquote du résidu anhydre à analyser de façon à avoir plus de o,5 mg de sucres dans la prise d'essai à laquelle on additionne exactement environ $\mathbf{m g}$ de méso-inositol. On ajoute alors successivement $x \mathrm{ml}$ de diméthylformamide, puis $0,5 \mathrm{ml}$ d'hexaméthyldisilazane et $0,5 \mathrm{ml}$ de triméthylchorosilane. Après avoir laissé reposer le mélange, on obtient deux phases liquides distinctes. Les dérivés triméthylsilylés se trouvent dans la phase supérieure. 


\section{Analyse chromatographique}

a) Injection : Prélever $\mathrm{I} \mu \mathrm{l}$ dans la phase surnageante du milieu réactionnel et injecter sur la colonne analytique.

b) Programmation de température : La température initiale est fixée à $\mathrm{r}_{4} \mathrm{O}^{\circ} \mathrm{C}$. Dès l'injection, la température est programmée à une vitesse de $0,5^{\circ} / \mathrm{mn}$ jusqu'à élution complète du pic de mésoinositol, puis à une vitesse de $2^{\circ} / \mathrm{mn}$ jusqu'à $320^{\circ} \mathrm{C}$.

Les temps de programmation doivent être établis avec précision par un étalonnage des colonnes, en injectant un mélange de sucres purs.

\section{Interprétation des résultats}

Le facteur de réponse $\mathrm{K}$ de chaque sucre par rapport au standard interne (méso-inositol) est déterminé à partir de quantités connues des sucres purs silylés dans les conditions décrites précédemment.

$$
K=\frac{\text { surface du sucre étudié }}{\text { surface du standard interne }} \times \frac{\text { poids du standard interne }}{\text { poids du sucre étudié }}
$$

On calcule la quantité des sucres étudiés par la formule :

Poids du sucre étudié $=\frac{\text { surface du sucre étudié }}{\text { surface du standard interne }} \times \frac{\text { poids du standard interne }}{\mathbf{K}}$

\section{JUSTIFICATTON DE LA MÉTHODE}

\section{Préparation des dérivés volatils}

Nous avons utilisé le diméthylformamide comme solvant des sucres au lieu de la pyridine pour deux raisons :

$\mathrm{I}^{\circ}$ Les monosaccharides se dissolvent très rapidement dans la pyridine (quelques minutes), mais la vitesse de dissolution des oligosaccharides et de l'inositol est beaucoup plus lente, ce qui rend difficile la détermination du temps optimum de silylation lorsque l'on est en présence d'un mélange de sucres inconnus. Ces phénomènes expliquent les temps de silylation très variables proposés par les différents auteurs qui ont utilisé la pyridine : de 5 minutes (BENTLEY et al., I963) à 12 heures (SWEELEY, I965). C'est la raison pour laquelle nous avons préféré utiliser le diméthylformamide qui permet une silylation complète des différents sucres au bout d'un temps déterminé. En effet, le diméthylformamide provoque la formation d'une phase appelée hexaméthyldisiloxane (HMDSO) qui présente une grande affinité pour les dérivés silylés de tous les sucres. Ces produits sont donc totalement dissous dans HMDSO qui compose la face surnageante du milieu réactionnel. Cependant, cette méthode est longue puisque la formation d'HMDSO commence 8 heures après le mélange des réactifs et se poursuit environ pendant $\mathrm{I} 8$ heures, mais elle permet de dissoudre quantitativement la totalité des sucres présents dans le mélange. De plus, la pyridine favorise les réactions de mutarotation (ISBFLL et al., I969) qui multiplient le nombre de pics obtenus pour un sucre donné, ce qui rend difficile l'interprétation des chromatogrammes d'un mélange de glucides; en revanche, le diméthylformamide modifie peu la structure des molécules de glucides présentes dans le mélange à analyser. 
$2^{\circ}$ L'utilisation du diméthylformamide permet d'évaluer les pentoses quantitativement et avec précision, bien qu'ils soient élués dans une zone très voisine de celle du pic de solvant. En effet, ELIIS (Ig69) a montré que le pic d'hexaméthyldisiloxane est beaucoup plus étroit et revient plus rapidement à la ligne de base que le pic de pyridine (fig. I et 2 ).

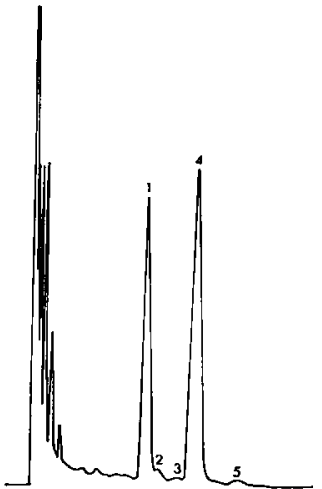

Frg. I. - Chromatogramme de glucides dissous dans le diméthylformamide

1. 2. 3. arabinose 4. 5. xylose



La méthode proposée s'applique à des échantillons anhydres. Cependant, nous avons constaté que certains produits très hygroscopiques s'humidifiaient pendant la pesée de l'échantillon précédant la silylation. Pour cette raison, nous avons augmenté les quantités d'hexaméthyldisiloxane et de triméthylchlorosilane dans le mélange réactionnel (BROBST et LOTT, I966) (fig. 3). On peut en plus ajouter aux réactifs de

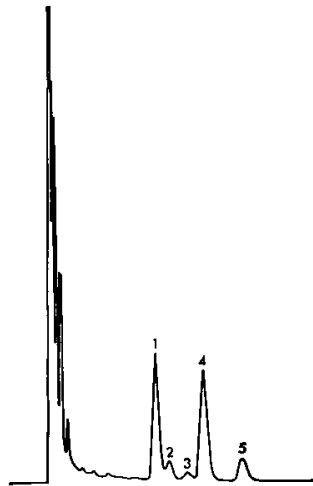

FIG. 3. - Chromatogramme de glucides silyles en milieu aqueux selon notre méthode

I. 2. 3. arabinose 4. 5. xylose

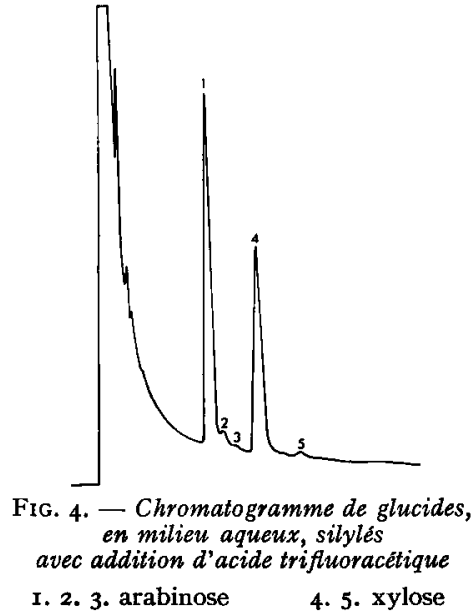

silylation $0,1 \mathrm{ml}$ d'acide trifluoracétique qui accélère la vitesse de formation des dérivés silylés et favorise la réaction en milieu humide (fig. 4). Dans ce cas, la phase d'HMDSO n'apparaît plus, ce qui provoque une traînée du pic de solvant sur le chromatogramme.

Annales de Biologie animale, -- 1972. 


\section{Choix de la colonne}

La séparation des pentoses, hexoses, di- et triholosides ne peut être réalisée qu'avec des colonnes ayant un important pouvoir de résolution. Elle nécessite par conséquent l'emploi de colonnes longues et de ce fait l'élution des triholosides ne se fera qu'à partir de $300^{\circ} \mathrm{C}$.

Nous avons fait des essais de séparation en utilisant différentes phases stationnaires : SE 30 (résine méthyl), SE 52 (résine méthyl-phényl), OVI (résine diméthyl) et OVI7 (résine phényl-méthyl). Le pouvoir de résolution de ces différentes phases est sensiblement le même. Cependant, OVI et OVI7 ont une température limite d'utilisation de $35^{\circ} \mathrm{C}$ tandis que $\mathrm{SE}, 30$ et $\mathrm{SE} 52$ se décomposent à $300^{\circ} \mathrm{C}$. Le choix entre OVI et OVI7 a été déterminé par le caractère non polaire d'OVI qui convient mieux au problème de la séparation des glucides (SWEELEY et al., I963).

\section{Choix du standard interne}

Le méso-inositol a été choisi comme standard interne car son pic d'élution n'interfère pas avec d'autres pics de glucides connus. Nous avons vérifié que ce composé n'est pas présent dans les milieux biologiques que nous nous proposons d'étudier.

\section{Programmation de température}

Il est difficile de donner avec précision un type de programmation de température car elle est liée aux caractéristiques du chromatographe et des colonnes qui ne sont jamais rigoureusement identiques. La température initiale étant fixée à $I 40^{\circ} \mathrm{C}$, nous utilisons les vitesses de programmation de $0,5^{\circ} / \mathrm{mn}$ puis de $2 \% / \mathrm{mn}$ jusqu'à $320^{\circ} \mathrm{C}$.

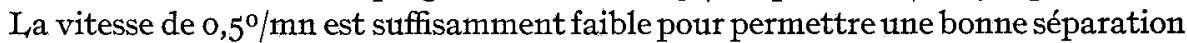
des pentoses et des hexoses tout en réduisant la durée de l'analyse. La vitesse de $2 \% / \mathrm{mn}$ permet d'accroître rapidement la température du four tout en séparant convenablement la majorité des di- et triholosides. Il est possible d'interrompre l'élévation de température au cours de la programmation afin d'améliorer la séparation de deux composés voisins.

\section{DISCUSSION}

\section{Validité de la méthode}

Cette méthode permet de séparer la majorité des oses simples ou méthylés, des di- et triholosides que l'on rencontre habituellement dans les milieux biologiques (fig. $5,6,7,8$ ). Des dosages comparatifs du glucose par chromatographie en phase gazeuse et par colorimétrie (dosage enzymatique du glucose par HILL et KESSLER, I96I) ont été effectués sur des hydrolysats de produits complexes (protozoaires du rumen). Nous avons ainsi vérifié que pour des teneurs en glucose variant de 0,4 à $30 \mathrm{p}$. Ioo de la matière sèche, les résultats obtenus par les deux méthodes étaient semblables $(r=0,994 ; n=44)$. Nous avons limité cette étude comparée au dosage du glucose à cause de la faible spécificité des méthodes colorimétriques pour les autres sucres. 
Nous avons effectué des essais de surcharge de glucose (environ $0,6 \mathrm{mg}$ ) sur des hydrolysats acides (par $\mathrm{H}_{2} \mathrm{SO}_{4} \mathrm{~N}$ à $100^{\circ} \mathrm{C}$ pendant 4 heures) et sur des hydrolysats enzymatiques (dosage de l'amidon, Thivend, Mercier et GuIl,Bot, I965) de proto-

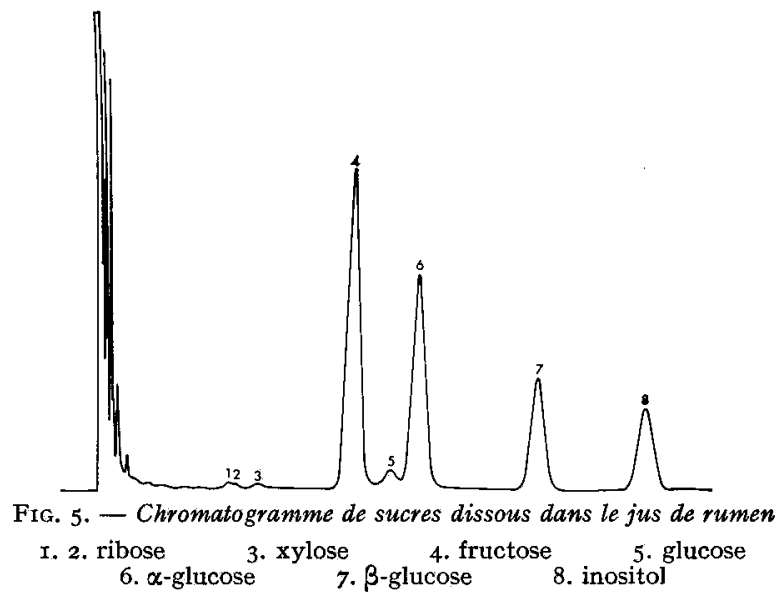

zoaires du rumen représentant environ $2 \mathrm{mg}$ de glucose dans la prise d'essai. La quantité de glucose ajoutée a été retrouvée à $\pm 3 \mathrm{p}$. Ioo près sur les hydrolysats enzymatiques et à $\pm 5 \mathrm{p}$. Ioo près sur les hydrolysats acides. L'augmentation de l'incertitude relative est due au fait que l'hydrolyse acide met en évidence différents pics de glu-

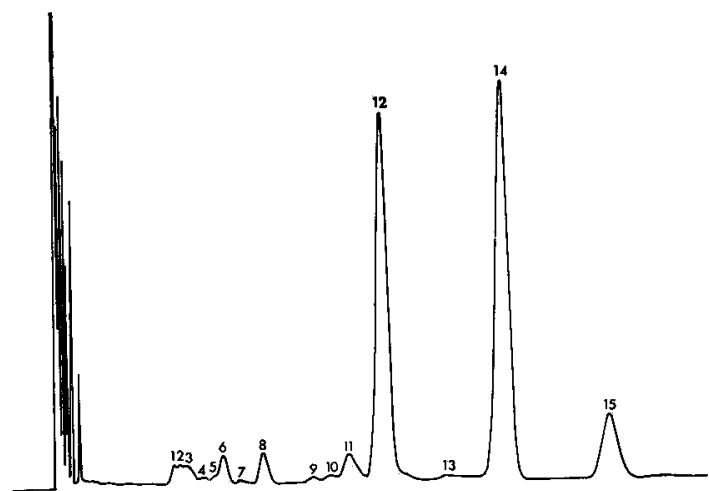

FIG. 6. - Chromatographie d'un hydrolysat acide de protozoaires du vumen neutralise par $B a(\mathrm{OH})_{2}$
r. 2. arabinose
3. 4. ribose
5. rhamnose
6. 8. xylose
7. 2. desoxyglucose
9. mannose

Io. galactosamine

Ir. glucosamine + galactose

I2. $\alpha$-glucose

13. acide galacturonique

I4. $\beta$-glucose

I5. inositol

cides proches du pic de glucose et, de ce fait, rend l'interprétation quantitative du chromatogramme plus délicate. Pour la même raison, les "surcharges " de mannose ont été retrouvées à $\pm 5 \mathrm{p}$. Ioo près. Cependant, il est difficile de séparer certains 
couples de sucres dans nos conditions expérimentales (fructose et mannose - arabinose et rhamnose - saccharose et lactose) et la durée d'analyse est relativement longue (de $60 \mathrm{mn}$ pour les pentoses et les hexoses à $\mathrm{I} 8 \mathrm{o} \mathrm{mn}$ pour les di-et triholosides). Pour résoudre ces deux problèmes, il sera sans doute nécessaire d'utiliser des colonnes capillaires qui permettent une séparation plus rapide des différents glucides et qui ont un pouvoir séparateur et un nombre de plateaux théoriques supérieurs à ceux des colonnes classiques.

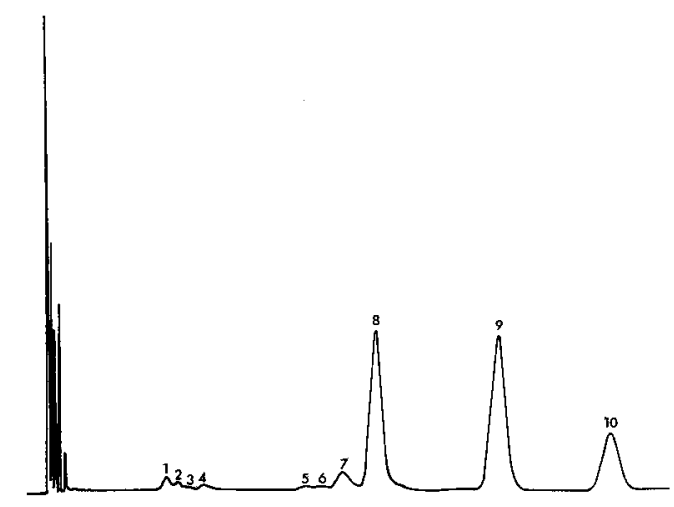

FIG. 7. - Chromatographie de bactéries du rumen hydrolysées par $\mathrm{H}_{2} \mathrm{SO}_{4} \mathrm{~N}$ à $100^{\circ} \mathrm{C}$ pendant 4 heures et neutralisées par $\mathrm{Ba}(\mathrm{OH})_{2} 0,3 \mathrm{~N}$
I. 4. rhamnose
2. 3. ribose
7. glucosamine + galactose
5. mannose
8. $\alpha$-glucose
6. galactosamine
9. $\beta$-glucos
Io. inositol

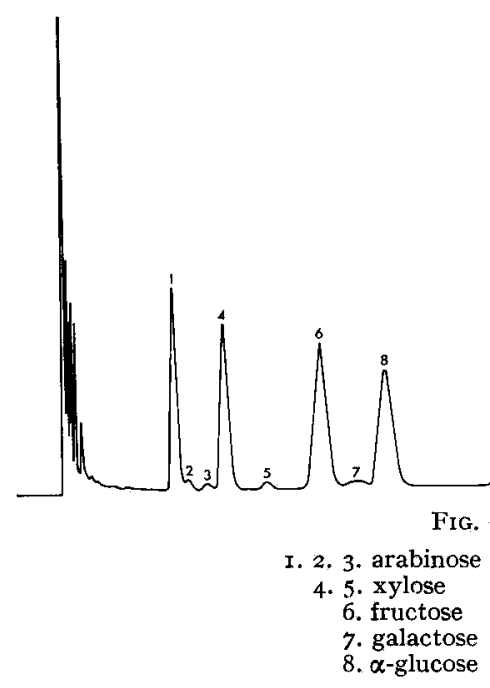

\section{Sensibilité et précision}

Cette méthode permet de déceler des quantités de glucides très faibles, de l'ordre de $I \gamma$, sans pouvoir toutefois les doser avec précision. Lorsque la quantité de glucides 
présente dans la prise d'essai est comprise entre roo $\gamma$ et $500 \gamma$, la précision de la méthode est environ de ro $\mathrm{p}$. I00; pour des quantités supérieures à $500 \gamma$, la précision varie de 3 à $8 \mathrm{p}$. roo selon la nature du glucide étudié.

Nous avons effectué divers essais de "répétabilité " qui ont porté, d'une part sur l'étude qualitative, et d'autre part sur l'étude quantitative d'un mélange de sucres; les sucres ont été identifiés par leur indice de rétention relatif ;

$$
d=\frac{d r-d m}{d i-d m} \text { où } d r-d m
$$

est la distance de rétention réduite du glucide considéré, di est la distance de rétention de l'inositol. Lorsque les conditions opératoires sont rigoureusement identiques, la "répétabilité " de l'indice de rétention relatif de chaque glucide est parfaite (tabl. 2 ).

TABI,EAU 2

Nombre de plateaux théoriques et indices de rétention relatifs de la colonne (1)

\begin{tabular}{|c|c|c|}
\hline Composé & $\begin{array}{c}\text { Nombre de } \\
\text { plateaux théoriques } \\
(d=\text { distance } \\
\text { de rétention } \\
w=\text { base du pic) } \\
n=16 \times \frac{d^{2}}{w^{2}}\end{array}$ & $\begin{array}{l}\text { Indice de } \\
\text { rétention relatif }\end{array}$ \\
\hline Arabinose $\ldots \ldots \ldots \ldots$ & 690 (1 er pic) & 3 pics : $0,21-0,23-0,26$ \\
\hline xylose $\ldots \ldots \ldots \ldots$ & 730 (1er pic) & 2 pics : $0,30-0,37$ \\
\hline Fructose $\ldots \ldots \ldots \ldots$ & 1010 & 0,47 \\
\hline Glucose- $\alpha \ldots \ldots \ldots \ldots$ & 1720 & 0,59 \\
\hline Glucose $-\beta \ldots \ldots \ldots$ & 2120 & 0,80 \\
\hline Inositol $\ldots \ldots \ldots \ldots$ & 3000 & 1,00 \\
\hline Gentiobiose $\ldots \ldots \ldots$ & 30900 & 1,84 \\
\hline Tréhalose .......... & 33700 & 2 pics : $1,75-1,81$ \\
\hline Raffinose . . . . . . . . & 48890 & 2,11 \\
\hline Mélézitose $\ldots \ldots \ldots \ldots$ & 50570 & 2,14 \\
\hline
\end{tabular}

(1) Ces valeurs ne sont données qu'à titre indicatif. Elles devront être établies pour chaque série de mesures.

La détermination du facteur de réponse des glucides est fonction du rendement de la réaction de " silylation ". Elle dépend donc de la nature du glucide considéré et de sa quantité (tabl. 3). La précision est maximum lorsque l'on a environ I mg de chaque glucide dans la prise d'essai. 


\section{TABLEAU 3}

Facteurs de réponse de différents glucides et écart type en fonction de la quantité de glucide présente dans la prise d'essai ( $\left.{ }^{1}\right)$

\begin{tabular}{|c|c|c|}
\hline Glucide & $\begin{array}{c}\text { Moyenne du } \\
\text { facteur de réponse }\end{array}$ & $\begin{array}{c}\text { Ecart type du } \\
\text { facteur de réponse }\end{array}$ \\
\hline Arabinose & 0,69 & $\begin{array}{l}0,08 a \\
0,02 b\end{array}$ \\
\hline Ribose & 0,72 & $\begin{array}{l}0,09 a \\
0,04 b\end{array}$ \\
\hline Xylose $(\alpha+\beta)$ & 0,67 & $\begin{array}{l}0,06 a \\
0,03 \quad b\end{array}$ \\
\hline Mannose & 0,68 & $\begin{array}{l}0,08 a \\
0,04 b\end{array}$ \\
\hline Fructose & 0,71 & $\begin{array}{l}0,07 a \\
0,04 b\end{array}$ \\
\hline Galactose & 0,72 & $\begin{array}{l}0,08 a \\
0,04 \quad b\end{array}$ \\
\hline Glucose & 0,69 & $\begin{array}{l}0,07 a \\
0,03 \quad b\end{array}$ \\
\hline Inositol & 1,00 & \\
\hline Saccharose & 0,67 & $\begin{array}{l}0,06 a \\
0,03 b\end{array}$ \\
\hline Lactose & 0,61 & $\begin{array}{l}0,08 a \\
0,05 b\end{array}$ \\
\hline Maltose & 0,60 & $\begin{array}{l}0,08 a \\
0,04 b\end{array}$ \\
\hline Cellobiose & 0,62 & $\begin{array}{l}0,09 a \\
0,06 \quad b\end{array}$ \\
\hline Raffinose & 0,39 & $\begin{array}{l}0,09 a \\
0,06 \quad b\end{array}$ \\
\hline Mélézitose & 0,42 & $\begin{array}{l}0,09 a \\
0,05 b\end{array}$ \\
\hline
\end{tabular}

$a:$ La quantité de glucide présente dans la prise d'essai est inférieure à $0,4 \mathrm{mg}$.

$b$ : La quantité de glucide présente dans la prise d'essai est supérieure à $0,5 \mathrm{mg}$.

(1) Ces valeurs devront être établies périodiquement. 


\section{RECOMMANDATIONS IMPORTANTES}

Cette méthode s'applique à l'analyse de glucides issus de milieux complexes tels que les aliments (céréales, fourrages...), les contenus digestifs (protozoaires, bactéries et jus de rumen...), les fèces et le sang.

La silylation peut être effectuée directement sur le produit brut mais elle sera meilleure si on extrait au préalable, par l'eau ou par 1'alcool, les sucres à analyser et si on élimine par défécation les protéines de la solution. Malgré ces précautions, l'apparition de la phase surnageante n'est pas toujours très nette. Une centrifugation de deux minutes à 5000 tours/mn permet une bonne séparation des deux phases et fait disparaître sur le chromatogramme la traînée due à la présence de diméthylformamide dans l'hexaméthyldisiloxane. Un lavage à l'eau du mélange réactionnel atténue encore la traînée du pic de solvant mais il modifie la silylation des glucides et provoque une réduction de la réponse du détecteur.

Après une série de quatre injections, il est nécessaire d'extraire le dépôt de silice qui se forme sur 1'électrode collectrice du détecteur au cours de la combustion des dérivés silylés. En effet, cette couche isolante provoque une saturation prématurée du détecteur, ce qui diminue considérablement la précision de la méthode.

Reçu pour publication en juillet 1971.

\section{SUMIMARY}

\section{GAS-LIQUID CHROMATOGRAPHY OF MONO-, DI- AND TRISACCHARIDES IN BIOLOGICAL, SAMPLES}

The method we propose was developed in order to simultaneously analyse and quantify the content of pentoses, hexoses, di- and trisaccharides either in free state, or obtained after hydrolyssis, in biological samples : e.g. feeds, and digesta and blood.

The sample to be analyzed was previously freeze-dried. It then underwant chemical treatment which transformed the carbohydrate hydroxyl groups into volatile O-Trimethylsilyl ethers.

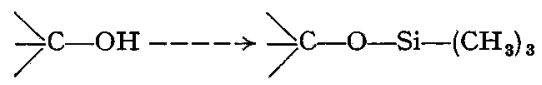

They were separated by gas-liquid chromatography using a chromatograph with a temperature programmer. The use of an internal standard (meso-inositol) allowed quantitative estimation of the various peaks obtained (table 3 ).

The modifications which we made to the classical methods of gas-liquid chromatography of carbohydrates were the following :

a) During the silylation reaction, the carbohydrates were dissolved, not in pyridine (SwEELEY et al., I963), but in dimethlyformamide (EllIS, I969). This allowed more exact quantitative results, especially when the pentoses were separated (fig. I and 2).

b) The quantities of reagents (hexamethyldisilazane and trimethylchlorosilane) were chosen so that it is now possible to analyze the carbohydrates, even if the sample is not perfectly anhydrous (fig. 3 and 4 ).

c) The stationary phase used was a nonpolar dimethyl silicone resin ( $\mathrm{OV}_{1}$ ) which has a high maximum temperature $\left(35^{\circ} \mathrm{C}\right)$, this allowing trisaccharide elution at $320^{\circ} \mathrm{C}$ (fig. 8)

Complete separation of most of the simple carbohydrates generally found in biological 
samples, may be obtained by this method (fig. 5, 6, 7 and 8). However, some carbohydrates are not well separated (arabinose-rhamnose, fructose-mannose, sucrose-lactose), and the analysis time is relatively long (from $60 \mathrm{mn}$ for pentoses and hexoses to $180 \mathrm{mn}$ for di- and trisaccharides). In order to solve these two problems, capillary columns will probably have to be used because they permit a more rapid separation of the various carbohydrates, and they have a separation capacity superior to that of classical columns (table 2).

\section{RÉFÉRENCES BIBLIOGRAPHIQUES}

Bentley R., Sweeley C. C., Makita M., Wells W. W., 1963. Gas chromatography of sugars and other polyhydroxy compounds. Biochem. Biophys. Res. Com., 11, 14-18.

Bishop C. T., Cooper F. P., 1960. Gas chromatography of some carbohydrates. Can. J. Chem., 38, $388-395$.

Brobst K. M., Lotr C. E., I966. Determination of some components in corn syrup by gas chromatography. Cereal Chem., 43, 35-43.

Clapperton J. F., Holliday A. G., I968. Estimation of carbohydrates in worts and beers by gas chromatography. J. Inst. Brew., 74, I64-I69.

ELLIS W. C., I969. Solvents for the formation and quantitative chromatography of trimethylsilyI derivatives of monosaccharides. J. Chromatog., 41, 325-334.

Hedgley E. G., Overend W. G., I960. Trimethylsilyl derivatives of carbohydrates. Chem. Ind. (Londres), 378-379.

Hill J. B., Kessler G., r96r. An automated determination of glucose utilizing a glucose-oxidaseperoxidase system. J. Lab. Clin. Med., 57, 970-980.

Isbell H. S., Frush H. L., WADE C. W. R., Hunter C. E., r969. Transformation of sugars in alkaline solutions. Carbohy-Res., 9, I63-I75.

Kimura M., Tohma M., Okasawa Y., Murrai N., I969, Determination of mono- and oligosaccharides in potato tubers by gas chromatography. J. Chromatog., 41, IIo-II2.

Mc Innes A. G., Ball D. M., Cooper F. P., Bishop C. T., I958. Separation of carbohydrates derivatives by gas-liquid chromatography. J. Chromatog., 1, 556-557,

Nunnikoven R., Ig68. Considération sur la conception d'un chromatographe en phase gazeuse moderne. Chromatographia, 1, 240-243.

OAtes M. D. G., Schrager J., 1967. The determination of sugars and amino sugars in the hydrolyzates of mucopolysaccharides by gas-chromatography. J. Chromatog., 28, 232-245.

SaWARdeker S. J., Sloneker J. H., Jeanes A., 1965. Quantitative determination of monosaccharides as their alditol-acetates, by gas-chromatography. Anal. Chem., 37, I602-I604.

Sweeley C. C., Bentley R., Makita M., Wells W. W., I963. Gas-liquid chromatography of trimethylsilyl derivatives of sugars and related substances. J. Am. Chem. Soc., 85, 2497-2507.

Sweeley C. C., I965. Analyse des hydrates de carbone par chromatographie en phase gazeuse. Bull. Soc. Chim. Biol., 47, I477-I493.

Thivend P., Mercier C., Guilbot A., 1965. Dosage de l'amidon dans les milieux complexes. Ann. Biol. anim. Bioch. Biophys., 5, 513-526.

Van Deemter J. J., ZuYderweg F. J., Klinkenberg A., 1956. In : Techniques de l'ingénieur. Mesures et analyse, p. 766-I, 768-3.

WELLS W. W., CHIN T., WEBER B., I964. Quantitative analysis of serum and urine sugars by gasliquid chromatography. Clin. Chim. Acta, 10, 352-359. 